\title{
Chronic Pain Treatment and Digital Health Era-An Opinion
}

\author{
V. Rejula, J. Anitha, R. V. Belfin* and J. Dinesh Peter \\ Department of Computer Science and Engineering, Karunya Institute of Technology and Sciences, Coimbatore, India
}

Keywords: chronic pain, health innovation, artificial intelligence, eHealth, digital health, virtual care, digital biomarkers, quality of life

\section{INTRODUCTION}

Pain is acknowledged as the body alarm system that reminds us of the environmental threat, muscle damage or the presence of some disease condition $(1,2)$. The pain is directly related to negative emotions (3). The stimulation of primary sensory neurons and specialized transduction machinery in their peripheral terminals is thought to be the physiological cause of pain. There are two sorts of pain based on the continuous pain period, namely acute and chronic (4).

Chronic pain is defined as pain that lasts for more than 3 months (5) and it is an significant health issue. Low back pain is one of the disease that wreaks more havoc on people's lives (68). Other leading causes and conditions of chronic pain are Rheumatoid arthritis (9-11), Shoulder Pain (8), Headache disorders (12, 13), Cancer (14, 15), Fibromyalgia (16, 17), Cervical and Thoracic Pain (18).

Depression (19, 20), anxiety (21), sleep problems (22), fatigue/lack of energy (23), and neurocognitive abnormalities (24) are all common comorbidities of chronic pain. These comorbidities reduce the patient's quality of life, create lost workdays, and make it difficult to maintain a healthy social life on their own $(25,26)$.

The relevance of chronic pain research along with the techniques and problems of diagnosing chronic pain, various treatment approaches, and current digital patient interaction tools has been discussed in this article. The summary of chronic pain treatment and digital patient engagement methods has been given in Figure 1. Finally, the benefits of digital health approaches during the COVID 19 pandemic and post-pandemic era are presented.

${ }^{*}$ Correspondence: R. V. Belfin researchbelfin@gmail.com

Specialty section: This article was submitted to

Digital Public Health,

a section of the journal

Frontiers in Public Health

Received: 18 September 2021 Accepted: 22 November 2021

Published: 10 December 2021

Citation:

Rejula V, Anitha J, Belfin RV and

Peter JD (2021) Chronic Pain

Treatment and Digital Health Era-An

Opinion.

Front. Public Health 9:779328.

doi: 10.3389/fpubh.2021.779328

\section{PAIN IDENTIFICATION METHODS}

There are two main aspects to pain: intensity (magnitude) and unpleasantness (effect) (27). In terms of characteristics such as duration, frequency, location, and severity, reporting the pain is essential (28). The measurement of pain can be divided into three broad classifications. "Self-report" (29) is the primary way for pain measurement. There are various scales such as Verbal Rating Scales (VRS) (30), Numerical rating scales (NRS) (31), Visual Analog Scales (VAS) (32), Smiley- based Wong-Baker Scale (WBS) (33), Faces Pain Scales-Revised (FPS-R) (34) are used to get the selfreports from the chronic pain patients (35-38). The second way of measuring pain is the "Observe behavior and infer" method (39). This method has various scales like Neonatal Infant Pain Scale (NIPS) (40), Crying Requires Increased Vital Signs Expression Sleeplessness (CRIES) (41), Face, Legs, Activity, Cry, Consolability (FLACC) (41) for Infants and Toddlers, then Pain Assessment Checklist for Seniors with Limited Ability to Communicate (PACSLAC) (42), DOLOPLUS2 (43), Pain Assessment in Advanced Dementia Scale (PAINAD) (44) for elderly with dementia and Behavioral Pain Scale (BPS) (45), Critical Care Pain Observation Tool (CPOT) (46), Nonverbal Pain Scale (NVPS) (47) for ill and unconscious persons. The third classification of measuring pain is "Indirect Physiology." These methods use modalities like electroencephalogram (EEG) 


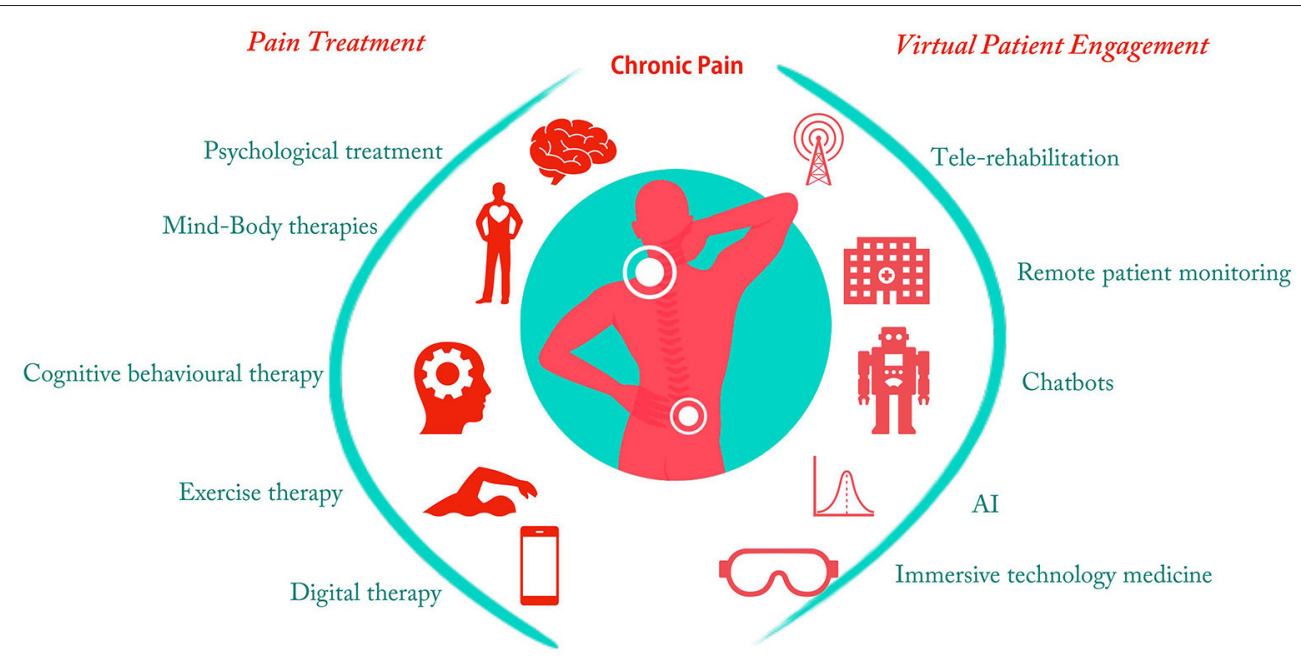

FIGURE 1 | Chronic pain treatment and digital patient engagement methods.

(48, 49), Magnetoencephalography (MEG) (49, 50), Positron emission tomography (PET) (7) and functional MRI (fMRI) (2, $51,52)$ to measure pain using the bio-markers. Having a variety of pain measurement methods does not mean that measuring pain is simple. There are many challenges in measuring pain accurately when we use self-reports and observe behavior and infer. The following section provides the challenges in measuring pain.

\subsection{Challenges of Measuring Pain}

The "self-report" and "Observe behavior and infer" report changes depending on mood, environment, and cognition. Even after the patients have been trained, the sensitivity, robustness, and reliability of the measurements obtained by self-report are quite poor. A baby, a demented old person, or an anesthetized or coma patient cannot self-report pain. One issue with observer rating is that not everyone exhibits pain reactions. A range of behavioral and physiological changes can be used to quantify responses to noxious stimuli. Lack of signals during noxious stimulation does not always imply the absence of pain. It's critical to have measurements that can be translated into pain treatment. Hence, there is a need for a bio-marker (35).

\subsection{Digital Bio-Markers}

A bio-marker is a feature that may be tested and assessed quantitatively as an indicator of a normal biological process, a pathological process, or the pharmacological (and nonpharmacological) response to a therapeutic treatment (53). Food and Drug Administration -National Institutes of Health (FDA$\mathrm{NIH}$ ) (54) working group classifies bio-markers into four types according to the development of drugs and biologic:

- Diagnostic bio-markers -To identify and validate the presence of pain.
- Prognostic bio-markers -To recognize the likelihood of a clinical event, disease recurrence, or progression in patients with disease of interest.

- Predictive bio-markers -To identify individuals who are more likely to have the bio-marker than those who do not.

- Pharmacodynamic bio-markers -To show that a biological response occurs in an individual exposed to a medical product.

\section{CHRONIC PAIN TREATMENT}

Chronic pain is treated in a variety of ways by healthcare practitioners. The treatment plan is determined by the type of pain, the cause (if known), and other factors that differ from person to person. Medication, lifestyle modifications, and treatments are all used in the best treatment programmes.

\subsection{Psychological Treatment}

Education is an essential element of psychological pain treatment (55) since it teaches patients how to handle a problematic condition. Talk therapy, Relaxation training, Stress management and pain coping skills training are the most frequent psychological treatments. You can obtain the assistance and counselling of a psychiatrist or psychologist through talk therapy. Cognitive therapy helps to alter the facts, ideas, and attitudes to change the amount of pain you feel. Relaxation training teaches you how to achieve a physiological state of profound relaxation that help you relieve pain. You may discover how your ideas impact your stress level and how to build a healthy response to difficult situations via stress management. You may learn how to adjust your life to the pain and have fun again through pain coping skills training (56).

\subsection{Mind-Body Therapies}

Several researchers on the use of mind-body therapy to treat persistent lower back pain have found that it is effective 
in reducing pain intensity, tension, and anxiety while also increasing aerobic ability. Mind-body treatments may encourage whole-body healing and include a fitness component to increase the brain's connection with physical function. They are often considered part of supplementary alternative or integrative medicine, which provides movement and energybased therapies. Patients with persistent lower back pain benefit from integrating complementary alternative mind-body treatments in their therapy, according to a variety of medical and healthcare professionals (e.g., primary care, spine surgeons, pain management). Holistic care is sometimes coupled with traditional medical therapy (57).

\subsection{Cognitive Behavioral Therapy}

Cognitive Behavioral Therapy (CBT) combines psychotherapy (talk therapy) with behavior therapy to help individuals better comprehend life's difficulties, retrain their attitudes, ideas, and perceptions, and develop problem-solving skills. A CBT therapist guides the person through goal-oriented therapy to learn how to manage stress, anxiety, depression, and sleep issues (58).

\subsection{Exercise Therapy}

When you're dealing with chronic pain or an injury, exercise therapy is the best option. It can help you feel and move better by making you stronger. Physical therapists are skilled in both treating and preventing pain. They will search for areas of weakness or stiffness that might be contributing to the tension in the painful regions. They'll also prescribe activities to help you move better and relieve discomfort in certain places (59).

\subsection{Digital Therapy}

The use of software, mobile applications, sensors, and other digital approaches as therapeutic interventions to address various medical problems is known as digital therapy. Digital therapies refer to all of these devices and methods together. In essence, digital treatment is not dissimilar to traditional faceto-face therapeutic procedures. The cognitive behavioral therapy technique is used in the majority of digital therapy practices. It focuses on the notion of providing frequent counseling to patients to improve their behavioral and lifestyle habits (60).

\section{CHRONIC PAIN VIRTUAL PATIENT ENGAGEMENT}

Many people believe that digital communication channels promote obsessive, compulsive social media illness in individuals. They are currently the effective means of contact between patients and medical professionals. We had no idea they'd become such a robust virtual tool for patient involvement. In these challenging days of the COVID-19, digital platforms assist in reaching out to patients regardless of location and save millions of lives.

\subsection{Telerehabilitation}

Telerehabilitation for pain treatment makes use of communication technologies to overcome geographical limitations. Access to such technology was indispensable during the COVID'19, and it was especially beneficial for individuals with chronic pain problems and unable to travel. Evaluating and treating such illnesses need a whole-health strategy that personalizes treatment choices and uses a biopsychosocial method to offer care. The care goals are the same as they are in a face-to-face encounter between a patient and a clinician. With proper consideration for staging a pain examination, a systematic approach to the physical visit, and the use of established clinical measures, telerehabilitation can be successfully applied in pain treatment (61).

\subsection{Remote Patient Monitoring}

RPM (remote patient monitoring) is a critical aspect of medical treatment in the future. When the objective of patient treatment is to enhance the quality of life, doctors must track how patients are doing between the appointments. This method of monitoring patients is especially crucial when dealing with chronic diseases like pain. The technologies like cloud computing, smartphone apps, sensors and wearables paved the way to improve this field (62). These software's collect, track, evaluate, and manage pain related data to improve the quality of life of a pain patient (54).

\subsection{Chatbots}

Today, algorithms-driven communications done through intelligent devices aim to treat hurts like substance use disorders, combat traumas, chronic pain and a worldwide pandemic. Conversational AI bots (chatbots) is a way to bridge the gap between the people and the knowledge they need and assisting them in completing tasks more quickly, all while utilizing existing tools. Conversational AI's future lies in incorporating it more into the care process across the whole care journey, whether it's assisting with you for appointments or just assisting you in living your best life $(63,64)$.

\subsection{Al}

The use of artificial intelligence in healthcare is growing, particularly in diagnosis and therapy management. AI applications in healthcare have recently made enormous impacts throughout medical services, igniting a debate about whether AI physicians would eventually displace human doctors (65). Experts, on the other hand, feel that human physicians will not be replaced by robots anytime soon. Artificial intelligence in healthcare, on the other hand, can assist doctors in making better clinical decisions or potentially replace human judgement in certain sectors of the industry.

Recent research used artificial intelligence, or machine learning algorithms, to physiological information from individuals with chronic pain, including respiration rate, oxygen levels, pulse rate, body temperature, blood pressure, and so on. The investigators' technique outperformed baseline models in determining subjective pain levels and differentiating between pain changes and irregular pain fluctuations (66).

\subsection{Immersive Technology Medicine}

A virtual reality (VR)-based digital therapy for persons with chronic pain has shown promising results in a clinical study (67), helping patients control their fear of movement, which 
can limit their activity and slow healing. People with chronic pain can utilize a virtual reality headset to lead them through a sequence of cognitive behavioral therapy (CBT) workouts to give them confidence in their fear of movement, commonly known as kinesiophobia (68).

\section{DISCUSSION}

The techniques for identifying, treating, and managing chronic pain had been discussed in the preceding section. People nowadays utilize the internet and other online resources to learn about the symptoms, treatments, and comorbidities of any ailments they may be suffering. For any disease, taking the medication without first visiting a doctor is not recommended. However, the utilization of digital health data can aid medical professionals, and scientists make better informed and effective decisions.

As mentioned in previous sections, chronic pain can be selfmanaged by adequate patient education, lifestyle therapies such as exercise, and weight loss activities. There are various hurdles to sufficient treatment in certain underdeveloped countries, such as geography, a lack of physicians, expense, inconvenience, impairment, or, more recently, COVID'19 limitations. However, given the widespread use of computers, intelligent applications, and the world wide web, digital health methods provide the possibility of delivering lifestyle therapies to individuals with chronic pain remotely and assisting them in self-management.

As people go about their regular lives, cell phones can collect digital traces. The behavioral characteristics of a person may be derived from this raw digital data. This digital data gives us the number of unique goals visited. For example, the average level of ambient noise detected during the night, and the sleep quality data offer information on the behavior patterns of a particular individual in a real-world scenario. Combining smartphone and wearable sensors may enable passive and remote real-time monitoring of changing symptoms by capturing behavioral and physiological changes during pain. Applying machine learning classification to smartphone data and other sensor data can help us classify chronic pain patients from the controls.

In healthcare, AI has a variety of effects. In most cases, AI in healthcare uses a web data set to give doctors and specialists access to a large number of diagnostic tools. Because doctors are extremely skilled in their area and keep up with current research, AI technology in healthcare creates a much faster outcome that can be matched with their clinical expertise.

The possibilities for AI applications in healthcare are vast, ranging from emergency rooms to general care to home care. Artificial intelligence can be used in healthcare to automate patient evaluation and minimise assessor bias. It can assess patient risk, analyse sickness (for example, by decoding ECG

\section{REFERENCES}

1. Davis KD, Flor H, Greely HT, Iannetti GD, MacKey S, Ploner M, et al. Brain imaging tests for chronic pain: medical, legal and ethical issues and recommendations. Nat Rev Neurol. (2017) 13:624-38. doi: $10.1038 /$ nrneurol.2017.122 findings and X-ray images), choose the best therapy based on a patient's clinical history and clinical trial results, track disease and detect early warning signals of worsening.

A biomarker is a biological indicator of an ailment or disease's presence. Biomarkers assist doctors in assessing and diagnosing patients as well as monitoring their therapeutic efficacy. Body temperature for fever, blood pressure for hypertension, and cholesterol levels for heart problems are examples of biomarkers. Doctors can provide target treatment by identifying and using pain biomarkers in conjunction with digital health technology. There are some drawbacks to using biomarkers for pain. Some pain biomarkers $(35,52,69-72)$ are stronger predictors in males, whereas others are better predictors of pain in women concerning gene expression. CNTN1 has been related to chronic pain in women, whereas LY9 (lymphocyte antigen 9) and MFAP3 have been connected to PTSD in males. We will have higher precision when we tailor the health care application by gender since men and women have distinct biomarkers for pain in terms of gene expression. Biomarkers for pain can be identified in brain signal markers also.

Currently, 81 percent of the world's population owns a smartphone, which opens up new possibilities for remote health monitoring. The fast growth of computing technologies like mobile apps, cloud computing, blockchain, big data analytics, AI, AR \& VR helps build more intelligent applications in this domain. Clinical trials and guidelines often recommend pharmacotherapy, psychotherapy, integrative therapies, and invasive procedures as part of a personalized multi-modal, multidisciplinary treatment strategy.

Incorporating the doctors with customized multi-modal treatment strategies, historical health data, advanced software technologies, and biomarkers can help the pain patients' improve their lives. The availability of huge data to train prediction algorithms, which assist (rather than replace) human doctors, stimulate curiosity-based thinking, enable cooperation, and reduce routine chores, thereby enhancing patient care, will push the application of $\mathrm{AI}$ in healthcare. Digital health solutions that are data-driven have the potential to change the health care domain. Suppose these technologies could be sustainably given at scale, they could provide equal access to expert-level treatment to everyone, everywhere, decreasing the worldwide health and wellness gap.

\section{AUTHOR CONTRIBUTIONS}

VR, JA, and RVB devised the work, the main conceptual ideas and the proof outline. VR and JA worked out almost all of the technical details. VR, RVB, and JDP worked on the manuscript. All authors contributed to the article and approved the submitted version. resting-state fMRI to classify chronic pain conditions. Front Neurosci. (2019) 13:1313. doi: $10.3389 /$ fnins.2019.01313

3. Wiech K, Tracey I. The influence of negative emotions on pain: behavioral effects and neural mechanisms. Neuroimage. (2009) 47:987-94. doi: 10.1016/j.neuroimage.2009.05.059 
4. Wang S, Du J, Xi D, Shao F, Qiu M, Shao X, et al. Role of GABAAR in the transition from acute to chronic pain and the analgesic effect of electroacupuncture on hyperalgesic priming model rats. Front Neurosci. (2021) 15:727. doi: 10.3389/fnins.2021.691455

5. Bailey JF, Agarwal V, Zheng P, Smuck M, Fredericson M, Kennedy DJ, et al. Digital care for chronic musculoskeletal pain: 10,000 participant longitudinal cohort study. J Med Internet Res. (2020) 22:1-14. doi: 10.2196/18250

6. Ung H, Brown JE, Johnson KA, Younger J, Hush J, Mackey S. Multivariate classification of structural MRI data detects chronic low back pain. Cerebral Cortex. (2014) 24:1037-44. doi: 10.1093/cercor/bhs378

7. Shen W, Tu Y, Gollub RL, Ortiz A, Napadow V, Yu S, et al. Visual network alterations in brain functional connectivity in chronic low back pain: a resting state functional connectivity and machine learning study. Neuroimage Clin. (2019) 22:101775. doi: 10.1016/j.nicl.2019.101775

8. Hakala PT, Rimpelä AH, Saarni LA, Salminen JJ. Frequent computer-related activities increase the risk of neck-shoulder and low back pain in adolescents. Eur J Public Health. (2006) 16:536-41. doi: 10.1093/eurpub/ckl025

9. Abe N, Fujieda Y, Asou K, Karino K, Kono M, Narita H. RestingState functional connectivity of pain processing brain region associated with therapeutic response to biologics in rheumatoid arthritis and spondyloarthritis. (2021) p. 3-5.

10. Bennell K. Digital health approaches for the lifestyle management of osteoarthritis. Osteoarthr Cartil. (2021) 29:S8. doi: 10.1016/j.joca.202 1.02 .020

11. Barroso J, Wakaizumi K, Reis AM, Baliki M, Schnitzer TJ, Galhardo $\mathrm{V}$, et al. Reorganization of functional brain network architecture in chronic osteoarthritis pain. Hum Brain Mapp. (2021) 42:1206-22. doi: $10.1002 / \mathrm{hbm} .25287$

12. Kim S, Bae DW, Park SG, Park JW. The impact of Pain-related emotions on migraine. Sci Rep. (2021) 11:1-9. doi: 10.1038/s41598-020-80094-7

13. Santana L, Liu C. Experience of botulinum toxin a injections for chronic migraine headaches in a pediatric chronic pain clinic. J Pediatr Pharmacol Ther. (2021) 26:151-6. doi: 10.5863/1551-6776-26.2.151

14. Adam R, Bond CM, Burton CD, de Bruin M, Murchie P. Can-Pain-a digital intervention to optimise cancer pain control in the community: development and feasibility testing. Support Care Cancer. (2021) 29:759-69. doi: 10.1007/s00520-020-05510-0

15. Mercadante S, Portenoy RK. Understanding the chameleonic breakthrough cancer pain. Drugs. (2021) 81:411-8. doi: 10.1007/s40265-021-01466-5

16. Gilron I, Robb S, Tu D, Holden R, Towheed T, Ziegler D, et al. Double-blind, randomized, placebo-controlled crossover trial of alpha-lipoic acid for the treatment of fibromyalgia pain: The IMPALA trial. Pain. (2021) 162:561-8. doi: $10.1097 /$ j.pain.0000000000002028

17. Goebel A, Krock E, Gentry C, Israel MR, Jurczak A, Urbina CM, et al. Passive transfer of fibromyalgia symptoms from patients to mice. J Clin Invest. (2021) 131:e144201. doi: 10.1172/JCI144201

18. Price M, Ravanpay A, Daniels C. Recognition of prodromal cervical spondylotic myelopathy presenting in a US Veteran referred to chiropractic for acute thoracic pain: a case report. J Bodyw Mov Ther. (2021) 28:13-7. doi: 10.1016/j.jbmt.2021.07.041

19. Ayzenberg I, Richter D, Henke E, Asseyer S, Paul F, Trebst C, et al. Pain, depression, and quality of life in neuromyelitis optica spectrum disorder: a cross-sectional study of 166 AQP4 antibody-seropositive patients. Neurol Neuroimmunol Neuroinflammat. (2021) 8:1-13. doi: 10.1212/NXI.0000000000000985

20. Kremer M, Becker LJ, Barrot M, Yalcin I. How to study anxiety and depression in rodent models of chronic pain? Eur J Neurosci. (2021) 53:236-70. doi: 10.1111/ejn.14686

21. Li XH, Matsuura T, Xue M, Chen QY, Liu RH, Lu JS, et al. Oxytocin in the anterior cingulate cortex attenuates neuropathic pain and emotional anxiety by inhibiting presynaptic long-term potentiation. Cell Rep. (2021) 36:109411. doi: 10.1016/j.celrep.2021.109411

22. Sorrentino F, Petito A, Angioni S, D'Antonio F, Severo M, Solazzo MC, et al. Impact of anxiety levels on the perception of pain in patients undergoing office hysteroscopy. Arch Gynecol Obstet. (2021) 303:999-07. doi: 10.1007/s00404-020-05885-9

23. Eccles JA, Davies KA. The challenges of chronic pain and fatigue. Clin Med. (2021) 21:19. doi: 10.7861/clinmed.2020-1009
24. Longoria JN, Pugh NL, Gordeuk V, Hsu LL, Treadwell M, King AA, et al. Patient-reported neurocognitive symptoms influence instrumental activities of daily living in sickle cell disease. Am J Hematol. (2021) 96:1396-406. doi: 10.1002/ajh.26315

25. Tomlinson RM, Bax KC, Ashok D, McMurtry CM. Health-related quality of life in youth with abdominal pain: an examination of optimism and pain self-efficacy. J Psychosom Res. (2021) 147:110531. doi: 10.1016/j.jpsychores.2021.110531

26. Aviram J, Lewitus GM, Vysotski Y, Yellin B, Berman P, Shapira $A$, et al. Prolonged medical cannabis treatment is associated with quality of life improvement and reduction of analgesic medication consumption in chronic pain patients. Front Pharmacol. (2021) 12:1199. doi: $10.3389 /$ fphar.2021.613805

27. Nahman-Averbuch H, Hughes C, Hoeppli ME, White K, Peugh J, Leon E, et al. Communication of pain intensity and unpleasantness through magnitude ratings: influence of scale type, but not sex of the participant. medRxiv. (2021) doi: 10.1101/2021.06.24.21259433

28. Könning A, Rosenthal N, Brown D, Stahlschmidt L, Wager J. Severity of chronic pain in german adolescent school students: a cross-sectional study. Clin J Pain. (2021) 37:118-25. doi: 10.1097/AJP.0000000000000898

29. Ali SM, Lau WJ, McBeth J, Dixon WG, van der Veer SN. Digital manikins to self-report pain on a smartphone: a systematic review of mobile apps. Eur J Pain. (2021) 25:327-38. doi: 10.1002/ejp.1688

30. Lee HJ, Cho Y, Joo H, Jeon JY, Jang YE, Kim JT. Comparative study of verbal rating scale and numerical rating scale to assess postoperative pain intensity in the post anesthesia care unit: a prospective observational cohort study. Medicine. (2021) 100:e24314. doi: 10.1097/MD.0000000000024314

31. Shrestha D, Shrestha R, Grotle M, Nygaard ØP, Solberg TK. Validation of the nepali versions of the neck disability index and the numerical rating scale for neck pain. Spine. (2021) 46:E325. doi: 10.1097/BRS.0000000000003810

32. Crellin DJ, Harrison D, Santamaria N, Huque H, Babl FE. The psychometric properties of the visual analogue scale applied by an observer to assess procedural pain in infants and young children: an observational study. $J$ Pediatr Nurs. (2021) 59:89-95. doi: 10.1016/j.pedn.2021.01.018

33. Alizadeh Z, Paymard A, Khalili A, Hejr H. A systematic review of pain assessment method in children. Ann Trop Med Public Health. (2017) 10:847-7. doi: 10.4103/ATMPH.ATMPH_155_17

34. Balparda K, Herrera-Chalarca T, Cano-Bustamante M, Gómez-González T, Nicholls-Molina MA. Rasch development and validation of a new faces scale for measuring pain, and its comparison with a gold standard: the Balparda-Herrera Pain Scale. Pain Manag. (2021) 11:689-703. doi: $10.2217 /$ pmt-2021-0005

35. Tracey I, Woolf CJ, Andrews NA. Composite pain biomarker signatures for objective assessment and effective treatment. Neuron. (2019) 101:783-800. doi: 10.1016/j.neuron.2019.02.019

36. Werner P, Lopez-Martinez D, Walter S, Al-Hamadi A, Gruss S, Picard R. Automatic recognition methods supporting pain assessment: a survey. IEEE Trans Affect Comput. (2019) p. 1-22. doi: 10.1109/TAFFC.2019.2946774

37. Xu $\mathrm{X}$, Huang $\mathrm{Y}$. Objective pain assessment: a key for the management of chronic pain. F1000Research. (2020) 9:1-7. doi: 10.12688/f1000research.20441.1

38. Louwerse MD, Hehenkamp WJ, van Kesteren PJ, Lissenberg BI, Brölmann HA, Huirne JA. Electronic continuous pain measurement vs verbal rating scale in gynaecology: a prospective cohort study. Eur J Obstetr Gynecol Reprod Biol. (2021) 256:263-9. doi: 10.1016/j.ejogrb.2020.11.012

39. Lautenbacher S, Sampson EL, Pähl S, Kunz M. Which facial descriptors do care home nurses use to infer whether a person with dementia is in pain? Pain Med. (2017) 18:2105-15. doi: 10.1093/pm/pnw281

40. Desai A, Aucott S, Frank K, Silbert-Flagg J. Comparing N-PASS and NIPS: improving pain measurement in the neonate. Advances in Neonatal Care. (2018) 18:260-6. doi: 10.1097/ANC.0000000000000521

41. Beltramini A, Milojevic K, Pateron D. Pain assessment in newborns, infants, and children. Pediatr Ann. (2017) 46:e387-95. doi: 10.3928/19382359-20170921-03

42. Ruest M, Bourque M, Laroche S, Harvey MP, Martel M, Bergeron-Vézina K, et al. Can we quickly and thoroughly assess pain with the PACSLAC-II? A convergent validity study in long-term care residents suffering from dementia. Pain Manag Nurs. (2017) 18:410-7. doi: 10.1016/j.pmn.2017.05.009 
43. Rostad HM, Utne I, Grov EK, Puts M, Halvorsrud L. Measurement properties, feasibility and clinical utility of the Doloplus-2 pain scale in older adults with cognitive impairment: a systematic review. BMC Geriatr. (2017) 17:257. doi: 10.1186/s12877-017-0643-9

44. Fry M, Elliott R. Pragmatic evaluation of an observational pain assessment scale in the emergency department: the pain assessment in advanced dementia (PAINAD) scale. Australas Emerg Care. (2018) 21:131-6. doi: 10.1016/j.auec.2018.09.001

45. Rijkenberg S, Stilma W, Bosman RJ, van der Meer NJ, van der Voort PH. Pain measurement in mechanically ventilated patients after cardiac surgery: comparison of the behavioral pain scale (bps) and the critical-care pain observation tool (CPOT). J Cardiothorac Vasc Anesth. (2017) 31:1227-34. doi: 10.1053/j.jvca.2017.03.013

46. Dale CM, Prendergast V, Gélinas C, Rose L. Validation of the critical-care pain observation tool (CPOT) for the detection of oral-pharyngeal pain in critically ill adults. J Crit Care. (2018) 48:334-8. doi: 10.1016/j.jcrc.2018.09.024

47. Delwel S, Perez RS, Maier AB, Hertogh CM, de Vet HC, Lobbezoo F, et al. Psychometric evaluation of the orofacial pain scale for non-verbal individuals as a screening tool for orofacial pain in people with dementia. Gerodontology. (2018) 35:200-13. doi: 10.1111/ger.12339

48. Afrasiabi S, Boostani R, Masnadi-Shirazi MA, Nezam T. An EEG based hierarchical classification strategy to differentiate five intensities of pain. Expert Syst Appl. (2021) 180:115010. doi: 10.1016/j.eswa.2021.115010

49. Chang W, Wang $\mathrm{H}$, Yan G, Lu Z, Liu C, Hua C. EEG based functional connectivity analysis of human pain empathy towards humans and robots. Neuropsychologia. (2021) 151:107695. doi: 10.1016/j.neuropsychologia.2020.107695

50. Witjes B, Baillet S, Roy M, Oostenveld R, Huygen FJ, de Vos CC. Magnetoencephalography reveals increased slow-to-fast alpha power ratios in patients with chronic pain. Pain reports. (2021) 6:e928. doi: 10.1097/PR9.0000000000000928

51. Tétreault P, Mansour A, Vachon-Presseau E, Schnitzer TJ, Apkarian AV, Baliki MN. Brain connectivity predicts placebo response across chronic pain clinical trials. PLoS Biol. (2016) 14:e1002570. doi: 10.1371/journal.pbio.1002570

52. Reddan MC, Wager TD. Modeling pain using fMRI: from regions to biomarkers. Neurosci Bull. (2018) 34:208-15. doi: 10.1007/s12264-017-0150-1

53. Davis KD, Aghaeepour N, Ahn AH, Angst MS, Borsook D, Brenton A, et al. Discovery and validation of biomarkers to aid the development of safe and effective pain therapeutics: challenges and opportunities. Nat Rev Neurol. (2020) 16:381-400. doi: 10.1038/s41582-020-0362-2

54. Pathak YJ, Greenleaf W, Verhagen Metman L, Kubben P, Sarma S, Pepin B, et al. Digital health integration with neuromodulation therapies: the future of patient-centric innovation in neuromodulation. Front Digit Health. (2021) 3:618959. doi: 10.3389/fdgth.2021.618959

55. de C Williams AC, Fisher E, Hearn L, Eccleston C. Psychological therapies for the management of chronic pain (excluding headache) in adults. Cochrane Database Syst Rev. (2020) 11:CD007407. doi: 10.1002/14651858.CD007407.pub4

56. Darnall BD. Psychological Treatment for Patients With Chronic Pain. Washington, USA: American Psychological Association (2019).

57. Martel MO, Bruneau A, Edwards RR. Mind-body approaches targeting the psychological aspects of opioid use problems in patients with chronic pain: evidence and opportunities. Transl Res. (2021) 234:114-28. doi: $10.1016 /$ j.trsl.2021.02.013

58. Zambelli Z, Halstead EJ, Fidalgo AR, Dimitriou D. Good sleep quality improves the relationship between pain and depression among individuals with chronic pain. Front Psychol. (2021) 12:1555. doi: 10.3389/fpsyg.2021.668930

59. Bandak E, Boesen M, Bliddal H, Daugaard C, Hangaard S, Bartholdy $\mathrm{C}$, et al. The effect of exercise therapy on inflammatory activity assessed by MRI in knee osteoarthritis: secondary outcomes from a randomized controlled trial. Knee. (2021) 28:256-65. doi: 10.1016/j.knee.2020. 12.022

60. Badawy SM, Abebe KZ, Reichman CA, Checo G, Hamm ME, Stinson J, et al. Comparing the effectiveness of education versus digital cognitive behavioral therapy for adults with sickle cell disease: protocol for the cognitive behavioral therapy and real-time pain management intervention for sickle cell via mobile applications (CaR. JMIR Res Protoc. (2021) 10:e29014. doi: 10.2196/29014

61. McGeary D, McGeary C. Telerehabilitation for headache management. Phys Med Rehabil Clin. (2021) 32:373-91. doi: 10.1016/j.pmr.2021.01.005

62. Argüello Prada EJ. The internet of things (IoT) in pain assessment and management: an overview. Inform Med Unlocked. (2020) 18:100298. doi: 10.1016/j.imu.2020.100298

63. Hauser-Ulrich S, Künzli H, Meier-Peterhans D, Kowatsch T. A smartphonebased health care chatbot to promote self-management of chronic pain (SELMA): pilot randomized controlled trial. JMIR mHealth uHealth. (2020) 8:e15806. doi: 10.2196/15806

64. Belfin R, Shobana A, Manilal M, Mathew AA, Babu B. A graph based chatbot for cancer patients. In: 20195 th International Conference on Advanced Computing \& Communication Systems (ICACCS). Coimbatore: IEEE(2019). p. 717-21.

65. Meskó B, Görög M. A short guide for medical professionals in the era of artificial intelligence. npj Digit Med. (2020) 3:126. doi: 10.1038/s41746-020-00333-z

66. Panaggio MJ, Abrams DM, Yang F, Banerjee T, Shah NR. Can subjective pain be inferred from objective physiological data? Evidence from patients with sickle cell disease. PLoS Comput Biol. (2021) 17:e1008542. doi: 10.1371/journal.pcbi.1008542

67. Logan DE, Simons LE, Caruso TJ, Gold JI, Greenleaf W, Griffin A, et al. Leveraging virtual reality and augmented reality to combat chronic pain in youth: position paper from the interdisciplinary network on virtual and augmented technologies for pain management. J Med Internet Res. (2021) 23:e25916. doi: 10.2196/25916

68. Haisley KR, Straw OJ, Müller DT, Antiporda MA, Zihni AM, Reavis KM, et al. Feasibility of implementing a virtual reality program as an adjuvant tool for peri-operative pain control; results of a randomized controlled trial in minimally invasive foregut surgery. Complement Ther Med. (2020) 49:102356. doi: 10.1016/j.ctim.2020.102356

69. Su Q, Song Y, Zhao R, Liang M. A review on the ongoing quest for a pain signature in the human brain. Brain Sci Adv. (2019) 5:274-87. doi: 10.26599/BSA.2019.9050024

70. Woo CW, Chang LJ, Lindquist MA, Wager TD. Building better biomarkers: brain models in translational neuroimaging. Nat Neurosci. (2017) 20:365-77. doi: $10.1038 / \mathrm{nn} .4478$

71. van der Miesen MM, Lindquist MA, Wager TD. Neuroimaging-based biomarkers for pain: state of the field and current directions. PAIN Rep. (2019) 4:e751. doi: 10.1097/PR9.0000000000000751

72. Lamichhane B, Jayasekera D, Jakes R, Glasser MF, Zhang J, Yang C, et al. Multi-modal biomarkers of low back pain: a machine learning approach. Neuroimage Clin. (2021) 29:102530. doi: 10.1016/j.nicl.2020. 102530

Conflict of Interest: The authors declare that the research was conducted in the absence of any commercial or financial relationships that could be construed as a potential conflict of interest.

Publisher's Note: All claims expressed in this article are solely those of the authors and do not necessarily represent those of their affiliated organizations, or those of the publisher, the editors and the reviewers. Any product that may be evaluated in this article, or claim that may be made by its manufacturer, is not guaranteed or endorsed by the publisher.

Copyright (C) 2021 Rejula, Anitha, Belfin and Peter. This is an open-access article distributed under the terms of the Creative Commons Attribution License (CC BY). The use, distribution or reproduction in other forums is permitted, provided the original author(s) and the copyright owner(s) are credited and that the original publication in this journal is cited, in accordance with accepted academic practice. No use, distribution or reproduction is permitted which does not comply with these terms. 